\title{
Convex Homotopy
}

\author{
W. A. Horn \\ Institute for Basic Standards, National Bureau of Standards, Washington, D.C. 20234
}

(March 30, 1971)

\begin{abstract}
Given any two continuous, convex functions $f$ and $g$ on a compact, convex set with certain properties ("regular" convexity), it is shown that an infinite number of homotopies of $f$ and $g$ exist which are also convex. One such homotopy in particular (the "basic" homotopy) is shown to have nice monotonicity properties and can be used as a basis for constructing other homotopies. A method of constructing the basic homotopy is given for the case where the domain of $f$ and $g$ is a line segment. (Theorems are for normed linear spaces only.)
\end{abstract}

Key words: Convex functions; convex sets; homotopy.

\section{Introduction}

In a normed linear space, a set $C$ is called convex if $\lambda x+\bar{\lambda} y \epsilon C$ for every $x, y \epsilon C$ and every $\lambda$ with $0 \leqslant \lambda \leqslant 1$, where $\bar{\lambda}=1-\lambda$. (We shall use the notation of a bar over a quantity to denote 1 -(that quantity) throughout the rest of this paper.) A mapping $f$ of $C$ into the reals is called convex if

$$
f(\lambda x+\bar{\lambda} y) \leqslant \lambda f(x)+\bar{\lambda} f(y)
$$

for all $x, y \epsilon C$ and $0 \leqslant \lambda \leqslant 1$. Given any two continuous convex maps $f$ and $g$ on $C$, we define a map

$$
h: C \times[a, b] \rightarrow R,
$$

where $-\infty<a<b<\infty$ and $R$ is the real numbers, to be a convex homotopy of $(f, g)$ if $h$ is a continuous convex map and $h(x, a)=f(x), h(x, b)=g(x)$, for all $x \in C$. (Usually $a=0$ and $b=1$ in the definition of homotopy, but this definition will simplify notation and is equivalent to the usual definition.)

The question arises whether any two continuous convex maps $f$ and $g$ can be connected by a convex homotopy and, if so, how many such homotopies exist and what are their properties. The following gives an affirmative answer to the basic question for certain types of domain sets and shows that there are always an infinite number of such homotopies. Certain characteristics of a "basic" convex homotopy are also derived.

It will be noted in the following that proofs of continuity are, for the most part, independent of convexity, and it would be possible to construct a "not-necessarily-continuous" convex homotopy of two maps, using the following procedures, with much less stringent conditions on the maps and their domain. This observation might lead to inquiry about the existence of convex sets given a specification of part of their boundaries. This idea will not be pursued here.

The reader is also referred to an article by Victor Klee ${ }^{1}$ which contains results similar to those of theorems 1,2, and 4, without the concept of continuity.

AMS Subject Classification: Primary 5225, Secondary 5540.

'Klee, V. L., Jr., External structure of convex sets. II, Math. Zeitsch. Bd. 69, S. 90-104 (1958). 


\section{Existence; Basic and Other Homotopies}

We define a regularly convex set $C$ to be a convex set with the following property. For each $x \in C, \epsilon>0$, and $0 \leqslant \lambda \leqslant 1$, there exists $\delta(x, \epsilon, \lambda)>0$ such that if $y$ and $z$ are any points of $C$ for which

$$
x=\lambda y+\bar{\lambda} z
$$

then for any point $x^{\prime} \epsilon C$ with $\left\|x-x^{\prime}\right\|<\delta$ there exist points $y^{\prime}, z^{\prime} \epsilon C$ such that

$$
x^{\prime}=\lambda y^{\prime}+\bar{\lambda} z^{\prime}
$$

and $\left\|y-y^{\prime}\right\|<\epsilon,\left\|z-z^{\prime}\right\|<\epsilon$. Not all convex sets are regularly convex, as will be shown by an example later, although all of the usual ones (e.g., line segment, disk in the plane) seem to be.

Given any pair of continuous convex maps $f$ and $g$ on a compact, regularly convex subset $C$ of a normed linear space, let

se defined by

$$
H: C \times[0,1] \rightarrow R
$$

$$
H(x, r)=\inf \{r g(y)+\bar{r} f(z): y, z \epsilon C, x=r y+\bar{r} z\} .
$$

( $H$, of course, is dependent on $f$ and $g$, but this dependence will not be indicated unless necessary for clarity.)

Theorem 1: For $\mathrm{f}, \mathrm{g}$, and $\mathrm{C}$ as above, $\mathrm{H}$ is a convex homotopy of $(\mathrm{f}, \mathrm{g})$ on $\mathrm{C} \times[0,1]$. Furthermore, if $\mathrm{h}$ is any other such homotopy, then $\mathrm{h}(\mathrm{x}, \mathrm{r}) \leqslant \mathrm{H}(\mathrm{x}, \mathrm{r})$ for all $\mathrm{x} \in \mathrm{C}, \mathrm{r} \in[0,1]$.

Proof: First, it is clear that $H(x, 0)=f(x)$ and $H(x, 1)=g(x)$, by the definition of $H$. It remains to show that $H$ is convex and continuous.

Let $(x, r)$ and $(y, s)$ be any two points of $C \times[0,1]$, with $r$ and $s$ not both 0 or 1 . If $x_{1}, x_{2}, y_{1}$, and $y_{2}$ are any points of $C$ such that $x=r x_{1}+\bar{r} x_{2}$ and $y=s y_{1}+\bar{s} y_{2}$, then for any $\lambda$ with $0<\lambda<1$ we have

$$
\begin{aligned}
\lambda(x, r)+\bar{\lambda}(y, s)=(\lambda x+\bar{\lambda} y, \lambda r+\bar{\lambda} s) & =\left(\lambda r x_{1}+\lambda \bar{r} x_{2}+\bar{\lambda} s y_{1}+\bar{\lambda} s y_{2}, \lambda r+\bar{\lambda} s\right) \\
& =\left([\lambda r+\bar{\lambda} s] \frac{\lambda r x_{1}+\bar{\lambda} s y_{1}}{\lambda r+\bar{\lambda} s}+[\lambda \bar{r}+\bar{\lambda} \bar{s}] \frac{\lambda \bar{r} x_{2}+\bar{\lambda} \bar{s} y_{2}}{\lambda \bar{r}+\bar{\lambda} \bar{s}}, \lambda r+\bar{\lambda} s\right) \\
& =\left(\mu\left[a_{1} x_{1}+\bar{a}_{1} y_{1}\right]+\bar{\mu}\left[a_{2} x_{2}+\bar{a}_{2} y_{2}\right], \mu\right),
\end{aligned}
$$

where

$$
\begin{aligned}
& \mu=\lambda r+\bar{\lambda} s, \\
& a_{1}=\frac{\lambda r}{\mu}, \\
& a_{2}=\frac{\lambda \bar{r}}{\bar{\mu}} .
\end{aligned}
$$

Thus,

$$
\begin{aligned}
H(\lambda(x, r)+\bar{\lambda}(y, s)) & =H\left(\left(\mu\left[a_{1} x_{1}+\bar{a}_{1} y_{1}\right]+\bar{\mu}\left[a_{2} x_{2}+\bar{a}_{2} y_{2}\right], \mu\right)\right) \leqslant \mu g\left(a_{1} x_{1}+\bar{a}_{1} y_{1}\right)+\bar{\mu} f\left(a_{2} x_{2}+\bar{a}_{2} y_{2}\right) \\
& (\text { by the definition of } H) \\
& \leqslant \mu a_{1} g\left(x_{1}\right)+\mu \bar{a}_{1} g\left(y_{1}\right)+\bar{\mu} a_{2} f\left(x_{2}\right)+\bar{\mu} \bar{a}_{2} f\left(y_{2}\right)
\end{aligned}
$$

(by the convexity of $f$ and $g$ ) 


$$
\begin{aligned}
& =\lambda r g\left(x_{1}\right)+\bar{\lambda} s g\left(y_{1}\right)+\lambda \bar{r} f\left(x_{2}\right)+\bar{\lambda} \bar{s} f\left(y_{2}\right) \\
& =\lambda\left[r g\left(x_{1}\right)+\bar{r} f\left(x_{2}\right)\right]+\bar{\lambda}\left[\operatorname{sg}\left(y_{1}\right)+\bar{s} f\left(y_{2}\right)\right] .
\end{aligned}
$$

Since $x_{1}, x_{2}, y_{1}$ and $y_{2}$ were chosen arbitrarily, satisfying only the conditions

it follows that

$$
x=r x_{1}+\bar{r} x_{2} \text { and } y=s y_{1}+\bar{s} y_{2},
$$

$$
\begin{aligned}
H(\lambda(x, r)+\bar{\lambda}(y, s)) \leqslant & \inf \left\{\lambda\left(r g\left(x_{1}\right)+\bar{r} f\left(x_{2}\right)\right): x_{1}, x_{2} \epsilon C \text { and } x=r x_{1}+\bar{r} x_{2}\right\} \\
& +\inf \left\{\bar{\lambda}\left(s g\left(y_{1}\right)+\bar{s} f\left(y_{2}\right)\right): y_{1}, y_{2} \epsilon C \text { and } y=s y_{1}+\bar{s} y_{2}\right\}=\lambda H(x, r)+\bar{\lambda} H(y, s),
\end{aligned}
$$

proving convexity of $H$.

A proof of continuity of $H$ is as follows. First, we define a norm on $C \times[0,1]$ as the sum of the norms on $C$ and $[0,1]$.

That is,

$$
\|(x, r),(y, s)\|=\|x-y\|+|r-s| .
$$

Next, note if $C$ is regularly convex, then $C \times[0,1]$ is also regularly convex. For, given $(x, r) \epsilon C \times[0,1], 0 \leqslant \lambda \leqslant 1$, and $\epsilon>0$, if

$$
(x, r)=\lambda(y, s)+\bar{\lambda}(z, t),
$$

then there exists $\delta>0$ such that, if $x^{\prime} \epsilon C$ and $\left\|x-x^{\prime}\right\|<\delta$, then there exist $y^{\prime}$ and $z^{\prime}$ such that

$$
x^{\prime}=\lambda y^{\prime}+\bar{\lambda} z^{\prime},
$$

where $\left\|y-y^{\prime}\right\|<\epsilon / 2,\left\|z-z^{\prime}\right\|<\epsilon / 2$. Thus if

$$
\left\|(x, r)-\left(x^{\prime}, r^{\prime}\right)\right\|<\delta
$$

and, say, $s<r<t$, while $r^{\prime}>r$, we merely take

$$
t^{\prime}=t
$$

and

$$
s^{\prime}=s+\frac{r^{\prime}-r}{\lambda}
$$

(where $\delta$ is chosen small enough so that $r^{\prime}, s^{\prime} \epsilon[s, t]$ and $\left|s-s^{\prime}\right|<\epsilon / 2$ ). Of course, if $\lambda=0$ we take $s^{\prime}=s, t^{\prime}=r^{\prime}$. A similar construction is followed if $r^{\prime}<r$.

It is clear, then, that the points $\left(y^{\prime}, s^{\prime}\right)$ and $\left(z^{\prime}, t^{\prime}\right)$ satisfy the conditions

$$
\left(x^{\prime}, r^{\prime}\right)=\lambda\left(y^{\prime}, s^{\prime}\right)+\bar{\lambda}\left(z^{\prime}, t^{\prime}\right)
$$

$\left\|(y, s)-\left(y^{\prime}, s^{\prime}\right)\right\|<\epsilon,\left\|(z, t)-\left(z^{\prime}, t^{\prime}\right)\right\|<\epsilon$. Thus $C \times[0,1]$ is also regularly convex.

The proof of continuity of $H$ will be given first for points $(x, 0)$ and $(x, 1)$, and then for other points.

Given any point (say) $(x, 0)$, if $(y, s)$ is any point for which

$$
\|(x, 0)-(y, s)\|<\delta,
$$


for some $\delta>0$, then $s<\delta$. Let $u$ and $v$ be two points of $C$ for which

and

$$
y=s u+\bar{s} v,
$$

$$
H(y, s)=s g(u)+\bar{s} f(v) .
$$

(Such points exist by the compactness of $C$.) Then

or

$$
s(y-u)=\bar{s}(v-y),
$$

$$
\|v-y\|=\frac{s}{\bar{s}}\|y-u\| \leqslant 2 \delta D
$$

if $\delta<1 / 2$, where $D$ is an upper bound on the distance between pairs of points of $C$.

Thus,

$$
\begin{aligned}
|H(y, s)-H(x, 0)| & =|s g(u)+\bar{s} f(v)-f(x)| \\
& \leqslant s|g(u)|+\bar{s}|f(v)-f(y)|+\bar{s}|f(y)-f(x)|+s|f(x)| \rightarrow 0 \text { as } \delta \rightarrow 0 .
\end{aligned}
$$

Thus $H$ is continuous for each $(x, 0)$ (and also for each $(x, 1)$ ).

Now let $(x, r)$ be any point of $C \times[0,1]$. We will show that, if $\left\{\left(x_{n}, r_{n}\right)\right\}$ is a sequence converging to $(x, r)$, then

and

$$
H(x, r) \leqslant \lim \inf H\left(x_{n}, r_{n}\right),
$$

$\lim \sup H\left(x_{n}, r_{n}\right) \leqslant H(x, r)$.

First, let $\left\{\left(x_{n_{i}}, r_{n_{i}}\right)\right\}$ be any subsequence of $\left\{\left(x_{n}, r_{n}\right)\right\}$ such that

$$
H\left(x_{n_{i}}, r_{n_{i}}\right) \rightarrow \lim \inf H\left(x_{n}, r_{n}\right) .
$$

By the compactness of $C$, for each $\left(x_{n_{i}}, r_{n_{i}}\right)$ there exist points $y_{n_{i}}$ and $z_{n_{i}}$ in $C$ such that

$$
\begin{gathered}
x_{n_{i}}=r_{n_{i}} y_{n_{i}}+\bar{r}_{n_{i}} z_{n_{i}}, \\
H\left(x_{n_{i}}, r_{n_{i}}\right)=r_{n_{i}} g\left(y_{n_{i}}\right)+\bar{r} f\left(z_{n_{i}}\right) .
\end{gathered}
$$

By the compactness of $C \times[0,1]$, there exists a subsequence of $\left\{\left(x_{n_{i}}, r_{n_{i}}\right)\right\}$, which we assume for convenience to be $\left\{\left(x_{n_{i}}, r_{n_{i}}\right)\right\}$ itself, and points $y, z \in C$, such that

$$
\begin{aligned}
y_{n_{i}} & \rightarrow y, \\
z_{n_{i}} & \rightarrow z,
\end{aligned}
$$

while

But then

$$
x=\lim x_{n_{i}}=\lim \left(r_{n_{i}} y_{n_{i}}+\bar{r}_{n_{i}} z_{n_{i}}\right)=r y+\bar{r} z .
$$

$$
\begin{aligned}
H(x, r) \leqslant r g(y)+\bar{r} f(z) & =\lim \left(r_{n_{i}} g\left(y_{n_{i}}\right)+\bar{r}_{n_{i}} f\left(z_{n_{i}}\right)\right) \\
& =\liminf H\left(x_{n_{i}}, r_{n_{i}}\right)
\end{aligned}
$$

proving the first part. 
Now let $u$ and $v$ be two points of $C$ such that

$$
\begin{gathered}
x=r u+\bar{r} v, \\
H(x, r)=r g(u)+\bar{r} f(v) .
\end{gathered}
$$

(Such points exist by the compactness of $C$.) By the regular convexity of $C \times[0,1]$, given $\epsilon>0$ there exists $\delta>0$ such that for any point $(w, s)$ with

$$
\|(w, s)-(x, r)\|<\delta
$$

there exist points $\left(w_{1}, s_{1}\right)$ and $\left(u_{2}, s_{2}\right)$, such that

and

$$
(w, s)=r\left(w_{1}, s_{1}\right)+\bar{r}\left(w_{2}, s_{2}\right),
$$

$$
\begin{gathered}
\left\|\left(w_{1}, s_{1}\right)-(u, 1)\right\|<\epsilon, \\
\left\|\left(w_{2}, s_{2}\right)-(v, 0)\right\|<\epsilon .
\end{gathered}
$$

But the convexity of $H$ implies

$$
H(w, s) \leqslant r H\left(w_{1}, s_{1}\right)+\bar{r} H\left(w_{2}, s_{2}\right) .
$$

By the continuity of $H$ at $(u, 1)$ and $(v, 0)$,

$$
H(w, s) \leqslant r H(u, 1)+\bar{r} H(v, 0)+\eta=H(x, r)+\eta,
$$

where $\eta>0, \eta \rightarrow 0$ as $\epsilon \rightarrow 0$. Thus, for a given $\eta>0$, there exists $\delta>0$ such that

$$
H(w, s) \leqslant H(x, r)+\eta
$$

whenever $\|(w, s)-(x, r)\|<\delta$.

Applying this inequality to the sequence $\left\{\left(x_{n}, r_{n}\right)\right\}$, we have

$$
H\left(x_{n}, r_{n}\right) \leqslant H(x, r)+\eta,
$$

when $\left\|(x, r)-\left(x_{n}, r_{n}\right)\right\|<\delta$, and hence

Since

$$
\lim \sup H\left(x_{n}, r_{n}\right) \leqslant H(x, r) \text {. }
$$

$$
\lim \inf H\left(x_{n}, r_{n}\right) \leqslant \lim \sup H\left(x_{n}, r_{n}\right),
$$

it follows that

$$
H(x, r)=\lim H\left(x_{n}, r_{n}\right),
$$

and hence that $H$ is continuous on $C \times[0,1]$.

Finally, if $h$ is any other convex homotopy of $(f, g)$, then

$$
h(x, r) \leqslant r h(y, 1)+\bar{r} h(z, 0)=r g(y)+\bar{r} f(z)
$$

for any $y, z \epsilon C$ such that $x=r y+\bar{r} z$. Thus

$$
h(x, r) \leqslant H(x, r) \text {. }
$$


This completes the proof of theorem 1 .

We shall call the map $H$ the basic convex homotopy of $(f, g)$ on $C \times[0,1]$. A basic convex homotopy $H^{\prime}$ on $C \times[a, b]$ is defined by

$$
H^{\prime}(x, r)=H\left(x, \frac{r-a}{b-a}\right)
$$

At this point it is appropriate to give examples of convex sets which are not compact or not regularly convex, and show that the $H$ defined for a given $f$ and $g$ need not be continuous.

First, let $C$ be the set of points in the plane defined by

$$
C=\{(0,0)\} \cup\{(x, y): 0<x<1,-1<y<1\},
$$

and let $f(x, y)=y$ and $g(x, y)=-y$. Then for every $(x, 0)$ except $(0,0)$, we have

while

$$
H\left((x, 0), \frac{1}{2}\right)=\frac{1}{2} f(x,-1)+\frac{1}{2} g(x, 1)=-1
$$

$$
\begin{aligned}
H\left((0,0), \frac{1}{2}\right) & =\frac{1}{2} f(0,0)+\frac{1}{2} g(0,0) \\
& =0 .
\end{aligned}
$$

For the next example, let $C$ be the convex hull of the set of points in three-space

$$
C^{\prime}=\{(0,0,-1)\} \cup\{(0,0,1)\} \cup\left\{(x, y, 0): y=x^{2}, y \leqslant 1\right\} .
$$

Then $C$ is clearly the union of two convex cones, from $(0,0,-1)$ and $(0,0,1)$ respectively, to the set

$$
\left\{(x, y, 0): x^{2} \leqslant y \leqslant 1\right\}
$$

Let $f(x, y, z)=z$, while $g(x, y, z)=-z$. Again, for $x \neq 0$ we have

while

$$
\begin{aligned}
H\left(\left(x, x^{2}, 0\right), \frac{1}{2}\right) & =\frac{1}{2} f\left(x, x^{2}, 0\right)+\frac{1}{2} g\left(x, x^{2}, 0\right) \\
& =0
\end{aligned}
$$

$$
\begin{aligned}
H\left((0,0,0), \frac{1}{2}\right) & =\frac{1}{2} f(0,0,-1)+\frac{1}{2} g(0,0,1) \\
& =-1 .
\end{aligned}
$$

Next we show that the basic convex homotopy is by no means the only convex homotopy joining two convex maps. From this point onward in the discussion we shall use the term "convex map" to refer only to a continuous, convex map on a compact, regularly convex domain set.

THEOREM 2: Let $\mathrm{f}, \mathrm{g}$, and $\mathrm{h}$ be convex maps on $\mathrm{C}$ satisfying

$$
h(x) \leqslant H(x, r)
$$

for all $\mathrm{x} \epsilon \mathrm{C}$, where $\mathrm{H}$ is the basic convex homotopy of $(\mathrm{f}, \mathrm{g})$ and $\mathrm{r}$ is some constant such that $0<\mathrm{r}<1$. Let $\mathrm{H}_{1}$ and $\mathrm{H}_{2}$ be the basic convex homotopies of $(\mathrm{f}, \mathrm{h})$ and $(\mathrm{h}, \mathrm{g})$ on $\mathrm{C} \times[0, \mathrm{r}]$ and $\mathrm{C} \times[\mathrm{r}, 1]$, respectively. If $\mathrm{G}$ is the map defined by

and

$$
\mathrm{G}(\mathrm{x}, \mathrm{s})=\mathrm{H}_{1}(\mathrm{x}, \mathrm{s}), \quad 0 \leqslant \mathrm{~s} \leqslant \mathrm{r}
$$

$$
\mathrm{G}(\mathrm{x}, \mathrm{s})=\mathrm{H}_{2}(\mathrm{x}, \mathrm{s}), \quad \mathrm{r} \leqslant \mathrm{s} \leqslant 1,
$$


then $\mathrm{G}$ is convex and hence is a convex homotopy of (f, $\mathrm{g})$.

Proof: It is clear that if $(x, s)$ and $(y, t)$ are two points of $C \times[0,1]$ such that $s, t<r$ or $s, t>r$, then

$$
G(\lambda(x, s)+\bar{\lambda}(y, t)) \leqslant \lambda G(x, s)+\bar{\lambda} G(y, t)
$$

by the convexity of $H_{1}$ or $H_{2}$. It remains to show that the above inequality holds when $s$ and $t$ are on different sides of $r$.

Suppose $s<r$ and $t>r$. Let

$$
\begin{aligned}
m & =\frac{t-r}{t-s}, \\
p & =\frac{s}{r}, \\
q & =\frac{t-r}{\bar{r}} .
\end{aligned}
$$

If $x_{1}, x_{2}, y_{1}$, and $y_{2}$ are points of $C$ such that

and

$$
x=p x_{1}+\bar{p} x_{2}
$$

then we have

$$
y=q y_{1}+\bar{q} y_{2},
$$

$$
\begin{aligned}
& m\left[p h\left(x_{1}\right)+\bar{p} f\left(x_{2}\right)\right]+\bar{m}\left[q g\left(y_{1}\right)+\bar{q} h\left(y_{2}\right)\right] \\
= & {\left[m \bar{p} f\left(x_{2}\right)+\bar{m} q g\left(y_{1}\right)\right]+m p h\left(x_{1}\right)+\bar{m} \bar{q} h\left(y_{2}\right) } \\
= & (m \bar{p}+\bar{m} q)\left[\bar{r} f\left(x_{2}\right)+r g\left(y_{1}\right)\right]+m p h\left(x_{1}\right)+\bar{m} \bar{q} h\left(y_{2}\right) \\
\geqslant & (m \bar{p}+\bar{m} q) h\left(\bar{r} x_{2}+r y_{1}\right)+m p h\left(x_{1}\right)+\bar{m} \bar{q} h\left(y_{2}\right)
\end{aligned}
$$

(by the fact that $h \leqslant H(\cdot, r)$ )

$$
\geqslant h\left([m \bar{p}+\bar{m} q]\left[\bar{r} x_{2}+r y_{1}\right]+m p x_{1}+\bar{m} \bar{q} y_{2}\right)
$$

(by the convexity of $h$ )

$$
\begin{aligned}
& =h\left(m \bar{p} x_{2}+\bar{m} q y_{1}+m p x_{1}+\bar{m} \bar{q} y_{2}\right) \\
& =h(m x+\bar{m} y) \\
& =G(m x+\bar{m} y, r) .
\end{aligned}
$$

Therefore, since $x_{1}, x_{2}, y_{1}$, and $y_{2}$ were arbitrary, satisfying only $x=p x_{1}+\bar{p} x_{2}$ and $y=q y_{1}+\bar{q} y_{2}$,

$$
\begin{aligned}
m G(x, s)+\bar{m} G(y, t)= & m \inf \left\{p h\left(x_{1}\right)+\bar{p} f\left(x_{2}\right): x_{1}, x_{2} \epsilon C, x=p x_{1}+\bar{p} x_{2}\right\} \\
+ & \bar{m} \inf \left\{q g\left(y_{1}\right)+\bar{q} h\left(y_{2}\right): y_{1}, y_{2} \epsilon C, y=q y_{1}+\bar{q} y_{2}\right\} \\
& \quad(\text { see definition after theorem 1) } \\
\geqslant & G(m x+\bar{m} y, r),
\end{aligned}
$$

noting $m s+\bar{m} t=r$. 
This establishes the convexity condition on $(x, s)$ and $(y, t)$ for the critical point $\lambda=m$, where the second coordinate becomes $r$. To prove it for any other $\lambda$, let us take (say) $0<\lambda<m$. Then

$$
\begin{aligned}
\lambda G(x, s)+\bar{\lambda} G(y, t) & =\frac{\lambda}{m}[m G(x, s)+\bar{m} G(y, t)]+\left(1-\frac{\lambda}{m}\right) G(y, t) \\
& \geqslant \frac{\lambda}{m} G(m(x, s)+\bar{m}(y, t))+\left(1-\frac{\lambda}{m}\right) G(y, t) \\
& \geqslant G\left(\frac{\lambda}{m}[m(x, s)+\bar{m}(y, t)]+\left(1-\frac{\lambda}{m}\right)(y, t)\right)
\end{aligned}
$$

(by the convexity of $H_{2}$ )

$$
=G(\lambda(x, s)+\bar{\lambda}(y, t)) .
$$

A similar argument holds when $\lambda>m$.

This completes the proof of the convexity of $G$.

Corollary 3: If $\mathrm{h}=\mathrm{H}(\cdot, \mathrm{r})$ in theorem 2, then $\mathrm{G}=\mathrm{H}$.

Proof. Because $G$ is a convex homotopy of $(f, g)$, we have $G \leqslant H$, by theorem 1 . But because $G=H_{1}$ on $C \times[0, r]$ and $G=H_{2}$ on $C \times[r, 1]$, the basic convex homotopies on these sets, whereas $H$ is another homotopy of $(f, H(\cdot, r))$ on $C \times[0, r]$ and of $(H(\cdot, r), g)$ on $C \times[r, 1]$, we must have $H \leqslant G$.

Theorem 4: Let $\mathrm{f}_{0}, \mathrm{f}_{1}, \ldots, \mathrm{f}_{\mathrm{n}}$ be convex maps defined on the set $\mathrm{C}$, and $0=\mathrm{r}_{0}<\mathrm{r}_{1}<\ldots$ $<\mathrm{r}_{\mathrm{n}}=1$ be a set of numbers in the unit interval. Denote by $\mathrm{H}_{\mathrm{ij}}$ the basic convex homotopy of $\left(\mathrm{f}_{\mathrm{i}}, \mathrm{f}_{\mathrm{j}}\right)$ on $\mathrm{C} \times\left[\mathrm{r}_{\mathrm{i}}, \mathrm{r}_{\mathrm{j}}\right]$.

If

$$
f_{i}(x) \leqslant H_{i-1, i+1}\left(x, r_{i}\right)
$$

for all $\mathrm{x} \epsilon \mathrm{C}$ and $\mathrm{i}=1,2, \ldots, \mathrm{n}-1$, then there exists a convex homotopy $\mathrm{G}$ on $\mathrm{C} \times[0,1]$ such that

for all $\mathrm{x} \epsilon \mathrm{C}$.

$$
\mathrm{f}_{\mathrm{i}}(\mathrm{x})=\mathrm{G}\left(\mathrm{x}, \mathrm{r}_{\mathrm{i}}\right)
$$

Proof: Let $G(x, r)=H_{i, i+1}(x, r)$ for $r_{i} \leqslant r \leqslant r_{i+1}$. The proof is by induction on $n$.

First, for $n=2$, the theorem is true by theorem 2. Now assume that the theorem has been proved for $n=2,3, \ldots, N-1$. Let $(x, r)$ and $(y, s)$ be any points in $C \times[0,1]$. In order to show convexity for $n=N$, we must prove that

$$
G(\lambda(x, r)+\bar{\lambda}(y, s)) \leqslant \lambda G((x, r))+\bar{\lambda} G((y, s)),
$$

for $0<\lambda<1$. By the induction hypothesis, it is only necessary to consider the case where $r_{0} \leqslant r \leqslant r_{1}$ and $r_{N-1} \leqslant s \leqslant r_{N}$. To simplify notation, let

$$
g(t)=G(a x+\bar{a} y, t)
$$

where $r<t<s$ and $a$ is such that $t=a r+\bar{a} s$. Let

$$
p=\frac{r_{2}-r_{1}}{r_{2}-r}
$$

and

$$
q=\frac{s-r_{2}}{s-r_{1}}
$$


Then

and

$$
g\left(r_{1}\right) \leqslant p g(r)+\bar{p} g\left(r_{2}\right)
$$

$$
g\left(r_{2}\right) \leqslant q g\left(r_{1}\right)+\bar{q} g(s),
$$

by the induction hypothesis. Multiplying the first inequality by $1 /(1-q+p q)$ and the second by $\bar{p} /(1-q+p q)$ and adding, we have

or

$$
g\left(r_{1}\right) \leqslant \frac{p g(r)}{1-q+p q}+\frac{\bar{q} \bar{p} g(s)}{1-q+p q},
$$

$$
g\left(r_{1}\right)=g(\nu r+\bar{\nu} s) \leqslant \nu g(r)+\bar{\nu} g(s),
$$

where

$$
\nu=\frac{p}{1-q+p q},
$$

proving the convexity inequality for $\lambda=\nu$. To prove the inequality for other values of $\lambda$, we use the same technique which was applied in theorem 2 . Thus if $\lambda<\nu$, for instance, we write

$$
\begin{aligned}
\lambda g(r)+\bar{\lambda} g(s) & =\frac{\lambda}{\nu}(\nu g(r)+\bar{\nu} g(s))+\left(1-\frac{\lambda}{\nu}\right) g(s) \\
& \geqslant \frac{\lambda}{\nu} g\left(r_{1}\right)+\left(1-\frac{\lambda}{\nu}\right) g(s) \\
& \geqslant g\left(\frac{\lambda}{\nu} r_{1}+\left(1-\frac{\lambda}{\nu}\right) s\right) \\
& =g(\lambda r+\bar{\lambda} s)
\end{aligned}
$$

by using the convexity of $g$ on $\left[r_{1}, s\right]$. This completes the proof.

Theorem 4 shows a method of constructing a homotopy between two given maps which has certain desired intermediate values. We shall call a convex map $G$ on $C \times[0,1]$, as in the above, a piecewise basic convex homotopy if $G$ is the basic homotopy of some pair $\left(f_{i}, f_{i+1}\right)$ on each set $C \times\left[r_{i}, r_{i+1}\right]$, where $0=r_{0}<r_{1}<\ldots<r_{n}=1$. The next theorem shows that any convex homotopy of maps on a convex set can be approximated by a piecewise basic convex homotopy.

TheOrem 5: Let $\mathrm{f}$ and $\mathrm{g}$ be any convex maps on the set $\mathrm{C}$, and let $\mathrm{G}$ be a convex homotopy of $(\mathrm{f}, \mathrm{g})$ on $\mathrm{C} \times[0,1]$. Then there exist piecewise basic convex homotopies $\mathrm{G}_{1}, \mathrm{G}_{2}, \ldots$., such that $\mathrm{G}_{\mathrm{i}} \rightarrow \mathrm{G}$ uniformly.

Proof: Let $M_{i}=\left\{0, r_{1}, r_{2}, \ldots, r_{m}=1\right\}$ be finite subsets of the unit interval, where mesh $\left(M_{i}\right) \rightarrow 0$. For any $i$, if $r_{j}<r_{j+1}<r_{j+2}$ are any three neighboring points of $M_{i}$, we note that

$$
G\left(x, r_{j+1}\right) \leqslant H_{j, j+2}\left(x, r_{j+1}\right),
$$

where $H_{j, j+2}$ is the basic convex homotopy of $\left(G\left(\cdot, r_{j}\right), G\left(\cdot, r_{j+2}\right)\right)$ on $C \times\left[r_{j}, r_{j+2}\right]$. Thus if $G_{i}$ is defined to be the piecewise convex homotopy of theorem 4 with points $0=r_{0}<r_{1}<\ldots .<r_{m_{i}}=1$ and

$$
f_{j}=G\left(\cdot, r_{j}\right)
$$

then $G_{i}$ is indeed convex, by theorem 4 .

Now for any $\epsilon>0$, there exists $\delta>0$ such that

$$
\left|G(x, r)-G\left(x, r^{\prime}\right)\right|<\epsilon
$$


whenever $\left|r-r^{\prime}\right|<\delta$, for all $x \in C$, by the compactness of $C \times[0,1]$. By taking mesh $\left(M_{i}\right)<\delta$, we have, for any $r$ such that $r_{j} \leqslant r \leqslant r_{j+1}$,

$$
G(x, r) \leqslant G_{i}(x, r) \leqslant \lambda G\left(x, r_{j+1}\right)+\bar{\lambda} G\left(x, r_{j}\right),
$$

where $r=\lambda r_{j+1}+\bar{\lambda} r_{j}$. Thus, since

$$
\begin{aligned}
\left|G(x, r)-\left[\lambda G\left(x, r_{j+1}\right)+\bar{\lambda} G\left(x, r_{j}\right)\right]\right| & \leqslant \lambda\left|G(x, r)-G\left(x, r_{j+1}\right)\right|+\bar{\lambda}\left|G(x, r)-G\left(x, r_{j}\right)\right| \\
& <\lambda \epsilon+\bar{\lambda} \epsilon=\epsilon,
\end{aligned}
$$

we have

$$
\left|G(x, r)-G_{i}(x, r)\right|<\epsilon,
$$

proving the theorem.

Finally, we note that forming a basic convex homotopy is a continuous process in $f$ and $g$.

Given two convex maps $f$ and $g$, let $\mathscr{H}$ be the operator defined as: $\mathscr{H}(f, g)$ is the basic homotopy $H_{f y}$ of $(f, g)$ on $C \times[0,1]$, where $C$ is the domain set of $f$ and $g$. Then $\mathscr{H}$ maps pairs of functions on $C$ into functions on $C \times[0,1]$. We shall use the sup norm for all functions in the following.

THEOREM 6: The operator $\mathscr{H}$ which maps pairs of convex maps on a set $\mathrm{C}$ into their basic convex homotopy on $\mathrm{C} \times[0,1]$ is continuous.

Proof: For any two given pairs of maps $(f, g)$ and $\left(f^{\prime}, g^{\prime}\right)$ such that

$$
\begin{aligned}
& \left\|f-f^{\prime}\right\|<\delta \\
& \left\|g-g^{\prime}\right\|<\delta
\end{aligned}
$$

it follows that, if $x, y$, and $z$ are points of $C$ such that

$$
x=r y+\bar{r} z \quad(0 \leqslant r \leqslant 1),
$$

then

$$
\left|r g(y)+\bar{r} f(z)-\left[r g^{\prime}(y)+\bar{r} f^{\prime}(z)\right]\right| \leqslant r\left|g(y)-g^{\prime}(y)\right|+\bar{r}\left|f(z)-f^{\prime}(z)\right|<r \delta+\bar{r} \delta=\delta .
$$

From this it follows easily that

$$
\left|H_{f g}(x, r)-H_{f^{\prime} g^{\prime}}(x, r)\right| \leqslant \delta .
$$

\section{Monotonicity Properties of the Basic Homotopy}

THEOREM 7: Let $\mathrm{f}$ and $\mathrm{g}$ be convex maps of the set $\mathrm{C}$, and $\mathrm{H}$ the basic convex homotopy of ( $\mathrm{f}, \mathrm{g})$ on $\mathrm{C} \times[0,1]$. If $\mathrm{f} \leqslant \mathrm{g}(\mathrm{f} \geqslant \mathrm{g})$ on $\mathrm{C}$, then $\mathrm{H}(\mathrm{x}, \cdot)$ is monotonic increasing (decreasing) on $[0,1]$, with strict monotonicity holding if strict inequality holds between $\mathrm{f}$ and $\mathrm{g}$.

Proof. Suppose $f \leqslant g$. By corollary 3 it is sufficient to show that

$$
f(x) \leqslant H(x, r) \leqslant g(x)
$$

for all $x \in C$ and $0 \leqslant r \leqslant 1$. Now

$$
\begin{aligned}
H(x, r) & \leqslant r g(x)+\bar{r} f(x) \\
& \leqslant r g(x)+\bar{r} g(x)=g(x)
\end{aligned}
$$


proving half of the inequality. Also if

$$
x=r y+\bar{r} z, \quad y, z \in C,
$$

then

$$
\begin{aligned}
r g(y)+\bar{r} f(z) & \geqslant r f(y)+\bar{r} f(z) \\
& \geqslant f(r y+\bar{r} z) \\
& =f(x)
\end{aligned}
$$

so that

$$
H(x, r) \geqslant f(x)
$$

A similar proof holds if $f \geqslant g$.

To prove strict monotonicity for (say) the case $f<g$, let

$$
\delta=\inf \{g(x)-f(x): x \in C\} .
$$

Then $\delta>0$, by the compactness of $C$. In the above argument we have

$$
\begin{aligned}
r g(y)+\bar{r} f(z) & \geqslant r \delta+r f(y)+\bar{r} f(z) \\
& \geqslant r \delta+f(x)
\end{aligned}
$$

implying $H(x, r)>f(x)$ if $r>0$.

Next we consider monotonicity in the maps $f$ and $g$ by taking their domain to be a subinterval of the real line.

THEOREM 8: Let $\mathrm{f}$ and $\mathrm{g}$ be convex maps defined on a compact subinterval $\mathrm{C}$ of the real line, and let $\mathrm{H}$ be the basic convex homotopy of $(\mathrm{f}, \mathrm{g})$ on $\mathrm{C} \times[0,1]$. If $\mathrm{f}$ and $\mathrm{g}$ are both monotonic increasing (decreasing) on $\mathrm{C}$, then $\mathrm{H}(\cdot, \mathrm{r})$ is also monotonic increasing (decreasing) on $\mathrm{C}$. Furthermore, strict monotonicity of $\mathrm{f}$ and $\mathrm{g}$ implies strict monotonicity of $\mathrm{H}(\cdot, \mathrm{r})$.

Proof. Consider two points $\left(x_{1}, r\right)$ and $\left(x_{2}, r\right)$, with $x_{2}>x_{1}$. If

$$
x_{2}=r y_{2}+\bar{r} z_{2}
$$

for some $y_{2}, z_{2} \epsilon C$, then clearly it is possible to find $y_{1}$ and $z_{1}$ in $C$ such that $y_{1} \leqslant y_{2}, z_{1} \leqslant z_{2}$, and

$$
x_{1}=r y_{1}+\bar{r} z_{1} .
$$

Thus

$$
r g\left(y_{2}\right)+\bar{r} f\left(z_{2}\right) \geqslant \operatorname{rg}\left(y_{1}\right)+\bar{r} f\left(z_{1}\right),
$$

by the monotonicity of $f$ and $g$. Since $y_{2}$ and $z_{2}$ were arbitrary, if follows that

$$
\inf \left\{r g\left(y_{2}\right)+\bar{r} f\left(z_{2}\right): y_{2}, z_{2} \epsilon C, x_{2}=r y_{2}+\bar{r} z_{2}\right\} \geqslant \inf \left\{r g\left(y_{1}\right)+\bar{r} f\left(z_{1}\right): y_{1}, y_{2} \epsilon C, x_{1}=r y_{1}+\bar{r} z_{1}\right\},
$$

or

$$
H\left(x_{2}, r\right) \geqslant H\left(x_{1}, r\right) \text {. }
$$


If $f$ and $g$ are strictly monotonic and $x_{2}-x_{1}=\delta>0$, then we note that $y_{2}-y_{1} \geqslant \delta$ or $z_{2}-z_{1} \geqslant \delta$ in the above. Now let

$$
\begin{aligned}
& a=\inf \{f(x)-f(x-\delta): x, x-\delta \epsilon C\} \\
& b=\inf \{g(x)-g(x-\delta): x, x-\delta \epsilon C\} .
\end{aligned}
$$

Then $a, b>0$, by the compactness of $C$ and the strict monotonicity assumption, while

$$
\left[r g\left(y_{2}\right)+\bar{r} f\left(z_{2}\right)\right]-\left[r g\left(y_{1}\right)+\bar{r} f\left(z_{1}\right)\right]=r\left[g\left(y_{2}\right)-g\left(y_{1}\right)\right]+\bar{r}\left[f\left(z_{2}\right)-f\left(z_{1}\right)\right] \geqslant \min \{r b, \bar{r} a\} .
$$

Thus

$$
H\left(x_{2}, r\right)-H\left(x_{1}, r\right) \geqslant \min \{r b, \bar{r} a\} .
$$

proving strict monotonicity.

It should be noted that the fact that $f(x) \geqslant g(y)$ for some $x$ and $y$ does not imply monotonicity of a convex homotopy $G$ of $(f, g)$ along the line joining $(x, 0)$ and $(y, 1)$, even if the conditions of theorems 7 and 8 are satisfied. For instance, if $f, g:[0,1] \rightarrow R$, and

$$
\begin{aligned}
& f(x)=1, \\
& g(x)=x,
\end{aligned}
$$

then $G(0,0)=G(1,1)=1$, but clearly

$$
\begin{aligned}
G\left(\frac{1}{2}, \frac{1}{2}\right) & \leqslant \frac{1}{2} G(0,1)+\frac{1}{2} G(1,0) \\
& =\frac{1}{2} .
\end{aligned}
$$

\section{Specific Forms of the Basic Convex Homotopy}

We have shown in theorems 2 and 4 that there are many convex homotopies connecting any given pair of convex maps. One interesting set of homotopies (because they can be written explicitly) is given in the following case. Let $f$ and $g$ be convex maps on $C$ such that $f-g=a$, a constant. If $h:[0,1] \rightarrow R$ is any convex map for which

$$
\begin{aligned}
& h(0)=0, \\
& h(1)=a,
\end{aligned}
$$

then the map $H: C \times[0,1] \rightarrow R$ defined by

$$
H(x, r)=f(x)+h(r)
$$

is a convex homotopy of $(f, g)$. In fact, if

$$
h(r)=r a,
$$

then $H$ is the basic homotopy. This raises some questions, about the form of the basic homotopy, which will now be considered. 
THEOREM 9: Let $\mathrm{f}$ and $\mathrm{g}$ be two convex, differentiable maps on a compact subinterval $\mathrm{C}$ of the real line. If $\mathrm{H}$ is the basic homotopy of $(\mathrm{f}, \mathrm{g})$ on $\mathrm{C} \times[0,1]$, then a necessary and sufficient condition that

$$
H(x, r)=f(x)+r(g(x)-f(x))
$$

is that $\mathrm{f}-\mathrm{g}$ is constant.

Proof. Consider any point $\left(x, \frac{1}{2}\right)$. Then

$$
H\left(x, \frac{1}{2}\right)=\frac{1}{2} f(x)+\frac{1}{2} g(x) .
$$

But if $\delta>0$, then we must have

$$
\frac{1}{2} H(x+\delta, 0)+\frac{1}{2} H(x-\delta, 1) \geqslant H\left(x, \frac{1}{2}\right),
$$

or

$$
\frac{1}{2} f(x+\delta)+\frac{1}{2} g(x-\delta) \geqslant \frac{1}{2} f(x)+\frac{1}{2} g(x) .
$$

Then,

$$
f(x+\delta)-f(x) \geqslant g(x)-g(x-\delta),
$$

implying

$$
f^{\prime}(x) \geqslant g^{\prime}(x)
$$

where $f^{\prime}$ and $g^{\prime}$ are the first derivatives of $f$ and $g$, respectively. Similarly, taking $\delta<0$, leads to

$$
f^{\prime}(x) \leqslant g^{\prime}(x)
$$

so that

$$
f^{\prime}(x)=g^{\prime}(x)
$$

Since $x$ was chosen arbitrarily, this implies that $f-g$ is constant, proving necessity. Sufficiency was shown above.

THEOREM 10. Let $\mathrm{f}$ and $\mathrm{g}$ be two convex maps on a set $\mathrm{C}$ such that, along any line segment in $\mathrm{C}$, $\mathrm{f}$ and $\mathrm{g}$ are differentiable except at, at most, a finite set of points. If $\mathrm{H}$ is the basic convex homotopy of $(\mathrm{f}, \mathrm{g})$ on $\mathrm{C} \times[0,1]$, then a necessary and sufficient condition that

$$
H(x, r)=f(x)+r(g(x)-f(x))
$$

is that $\mathrm{f}-\mathrm{g}$ is constant.

Proof. Consider any point $x \in C$. If $y$ is any other point of $C$, then by lemma $9 f-g$ is constant on the portions of the line segment $L$ joining $x$ and $y$ where $f$ and $g$ are both differentiable along $L$. By the continuity of $f$ and $g, f-g$ is therefore constant for all points on $L$, since there are at most a finite number of points at which the derivative along $L$ does not exist. Therefore,

$$
f(y)-g(y)=f(x)-g(x)=a .
$$

Since $y$ was arbitrary, the theorem is proved. 
Finally we show something about the explicit determination of $H$ for certain $f$ and $g$.

THEOREM 11. Let $\mathrm{f}$ and $\mathrm{g}$ be convex maps defined on a compact interval $\mathrm{C}$ of the real line, and let $\mathrm{H}$ be the basic convex homotopy of $(\mathrm{f}, \mathrm{g})$ on $\mathrm{C} \times[0,1]$. For any $(\mathrm{x}, \mathrm{r}) \in \mathrm{C} \times[0,1]$,

$$
H(x, r)=r g(y)+\bar{r} f(z),
$$

where $\mathrm{y}$ and $\mathrm{z}$ are points in $\mathrm{C}$ for which $\mathrm{x}=\mathrm{ry}+\overline{\mathrm{rz}}$ and $\mathrm{g}^{\prime}(\mathrm{y})=\mathrm{f}^{\prime}(\mathrm{z})$, if such points exist. If not, then $\mathrm{y}$ and $\mathrm{z}$ are such that $\mathrm{x}=\mathrm{ry}+\overline{\mathrm{r}} \mathrm{z}$ and

$$
\begin{aligned}
& f^{-}(z) \leqslant g^{+}(y), \\
& g^{-}(y) \leqslant f^{+}(z),
\end{aligned}
$$

where $\mathrm{f}^{-}, \mathrm{f}^{+}, \mathrm{g}^{-}, \mathrm{g}^{+}$are the left and right hand derivatives of $\mathrm{f}$ and $\mathrm{g}$, if such points exist. If neither of the above exist, then

$$
\mathrm{H}(\mathrm{x}, \mathrm{r})=\min \{\mathrm{rg}(\mathrm{y})+\overline{\mathrm{r}} \mathrm{f}(\mathrm{z}): \mathrm{x}=\mathrm{ry}+\overline{\mathrm{r}} \mathrm{z} \text { and } \mathrm{y} \text { or } \mathrm{z} \text { is an endpoint of } \mathrm{C}\} .
$$

Proof. Let

$$
h(y)=r g(y)+\bar{r} f\left(\frac{x-r y}{\bar{r}}\right)
$$

for all $y \in C$ for which $(x-r y) / \bar{r} \epsilon C$. Then $H(x, r)=\inf h(y)$. If there exists $y_{0} \epsilon C$ for which

$$
f^{-}\left(\frac{x-r y_{0}}{\bar{r}}\right) \leqslant g^{+}\left(y_{0}\right)
$$

and

then it is clear that, if $\delta>0$,

$$
g^{-}\left(y_{0}\right) \leqslant f^{+}\left(\frac{x-r y_{0}}{\bar{r}}\right)
$$

$$
\begin{aligned}
h\left(y_{0}+\delta\right) & =r g\left(y_{0}+\delta\right)+\bar{r} f\left(\frac{x-r y_{0}-r \delta}{\bar{r}}\right) \\
& \geqslant r\left(g\left(y_{0}\right)+\delta g^{+}\left(y_{0}\right)\right) \\
& +\bar{r}\left(f\left(\frac{x-r y_{0}}{\bar{r}}\right)-\frac{r \delta}{\bar{r}} f^{-}\left(\frac{x-r y_{0}}{\bar{r}}\right)\right) \\
& =r g\left(y_{0}\right)+\bar{r} f\left(\frac{x-r y_{0}}{\bar{r}}\right) \\
& +\delta r\left(g^{+}\left(y_{0}\right)-f^{-}\left(\frac{x-r y_{0}}{\bar{r}}\right)\right) \\
& \geqslant r g\left(y_{0}\right)+\bar{r} f\left(\frac{x-r y_{0}}{\bar{r}}\right)=h\left(y_{0}\right),
\end{aligned}
$$

whenever $h\left(y_{0}+\delta\right)$ exists. (If $h\left(y_{0}+\delta\right)$ does not exist, then either $y_{0}$ or $z_{0}=\left(x-r y_{0}\right) / \bar{r}$ is an endpoint of $C$.) Similarly,

Thus,

$$
h\left(y_{0}-\delta\right) \geqslant h\left(y_{0}\right) .
$$

$$
h\left(y_{0}\right)=\inf h(y)=H(x, r) .
$$


If there is no such point $y_{0}$, then for every $y$ in the interior of $C$ we have either

$$
f^{-}\left(\frac{x-r y}{\bar{r}}\right)>g^{+}(y)
$$

or

$$
g^{-}(y)>f^{+}\left(\frac{x-r y}{\bar{r}}\right) .
$$

Thus if neither $y$ nor $\frac{x-r y}{\bar{r}}$ is an endpoint of $C$, it is clear that there exists $\delta>0$ such that either

or

$$
h(y+\delta)<h(y)
$$

$$
h(y-\delta)<h(y)
$$

Since $h$ is continuous in $y$ and the domain of $h$ is a closed subinterval of $C$, it follows that $h$ must take its minimum value when $y$ or $\frac{x-r y}{\bar{r}}$ is an endpoint of $C$. This completes the proof.

(Paper 75B1\&2-343) 